\title{
Rapid magnetic flux variability on the flare star CN Leonis ${ }^{\star}$
}

\author{
A. Reiners ${ }^{1,2, \star \star}$, J. H. M. M. Schmitt ${ }^{1}$, and C. Liefke ${ }^{1}$ \\ 1 Hamburger Sternwarte, Gojenbergsweg 112, 21029 Hamburg, Germany \\ e-mail: [jschmitt;cliefke]@hs. uni-hamburg.de \\ 2 Universität Göttingen, Institut für Astrophysik, Friedrich-Hund-Platz 1, 37077 Göttingen, Germany \\ e-mail: Ansgar.Reiners@phys.uni-goettingen.de
}

Received 15 January 2007 / Accepted 28 February 2007

\section{ABSTRACT}

\begin{abstract}
We present UVES/VLT observations of the nearby flare star CN Leo covering the Wing-Ford FeH band near $1 \mu \mathrm{m}$ with high spectral resolution. Some of the $\mathrm{FeH}$ absorption lines in this band are magnetically sensitive and allow a measurement of the mean magnetic flux on CN Leo. Our observations, covering three nights separated by 48 hours each, allow a clear detection of a mean magnetic field of $B f \approx 2.2 \mathrm{kG}$. The differential flux measurements show a night-to-night variability with extremely high significance. Finally, our data strongly suggest magnetic flux variability on time scales as low as 6 hours in line with chromospheric variability.
\end{abstract}

Key words. stars: activity - stars: late-type - stars: magnetic fields - stars: individual: CN Leo

\section{Introduction}

Stellar activity can be observed in various tracers from all layers of a stellar atmosphere: Doppler imaging techniques reveal spots in the photosphere and trace regions of large magnetic flux, chromospheric emission shows up in specific lines like e.g. $\mathrm{H} \alpha$ or $\mathrm{Ca}$ II $\mathrm{H} \& \mathrm{~K}$ originating above photospherically active regions, and finally, X-rays trace the hottest material and allow the investigation and characterization of coronal properties. In active stars, these activity indicators can exceed the levels observed on the Sun by several orders of magnitude.

The strong activity observed in many stars implies strong magnetic fields, which are thought to lie at the root of all observed activity phenomena. Yet the direct measurement of magnetic fields in stellar photospheres is difficult. Most of the methods to measure magnetic fields require the detection of Zeeman broadening in magnetically sensitive spectral lines. Magnetic field strengths up to several kilo-Gauss and large filling factors have been measured for a few early $M$ dwarfs and T Tauri stars from optical Fe I and infrared Ti I lines (for an overview see Johns-Krull \& Valenti 2000). Spectropolarimetry allows to reconstruct the field geometry for some of these objects (Donati et al. 2006). However, appropriate atomic lines for such measurements either disappear or become more and more blended with molecular lines for objects later than spectral type mid M. Reiners \& Basri (2006) introduced a method to measure the mean magnetic flux of these ultracool dwarfs from lines of the $\mathrm{FeH}$ absorption band around $9950 \AA$. The FeH band comprises a large number of absorption lines with very different Zeeman sensitivity. Lines with small sensitivity can be used to determine the rotation velocity, and magnetically sensitive lines unambiguously indicate the quantity magnetic field strength $\times$ filling factor $(B f)$. Reiners \& Basri (2007) successfully measured the mean

* Based on observations collected at the European Southern Observatory, Paranal, Chile, 077.D-0011.

$\star \star$ Marie-Curie International Outgoing Fellow. magnetic field in a sample of $22 \mathrm{M}$ dwarfs with different activity levels and demonstrated that the magnetic flux of their sample objects is strongly correlated with $\mathrm{H} \alpha$ emission.

Stellar activity is inherently variable on time scales from seconds to decades. Variability on the shortest time scales occurs in the form of sudden outbursts or flares, which can be observed in several wavelength bands. While this variability has been extensively studied through proxy indicators like those mentioned above, we tackled the challenge to directly measure magnetic field changes and correlate the results with proxies like, e.g., $\mathrm{H} \alpha$ emission. For this purpose, we chose to study the flare star CN Leo (Gl 406). At a distance of 2.39 pc (Henry et al. 2004), $\mathrm{CN}$ Leo is one of the nearest late-type stars and also among the objects examined for magnetic fields by Reiners \& Basri (2007), who estimated an integrated surface magnetic flux of $B f \approx 2.4 \mathrm{kG}$. The temperature of CN Leo is $2800 \mathrm{~K}$ to $2900 \mathrm{~K}$ (Pavlenko et al. 2006; Fuhrmeister et al. 2005) at a spectral type of M5.5 or M6.0 (Reid et al. 1995; Kirkpatrick et al. 1991). Further information in particular on the coronal properties of CN Leo can be found in Fuhrmeister et al. (2007). CN Leo has - fortuitously but somewhat surprisingly - a rather low apparent rotation velocity of $v \sin i \approx 3.0 \mathrm{~km} \mathrm{~s}^{-1}$ (Mohanty \& Basri 2003; Reiners \& Basri 2007), thus making it an ideal target for spectroscopic high-resolution studies of stellar magnetic activity.

\section{Observations and data reduction}

CN Leo was observed during three half nights of May 19/20, 21/22 and May 23/24, 2006 with UVES/VLT. The UVES spectrograph was operated in dichroic mode with spectral coverage from $3200 \AA$ to $3860 \AA$ in the UV and from $6400 \AA$ to $10080 \AA$ with a gap between $8190 \AA$ and $8400 \AA$ in the red. We used a slit width of $1^{\prime \prime}$ resulting in a resolving power of $R \sim 40000$. Here we consider only the far red part of our spectra on the upper chip in the red arm. Exposure times varied according to seeing conditions from $100 \mathrm{~s}$ to $300 \mathrm{~s}$, resulting in a total of 


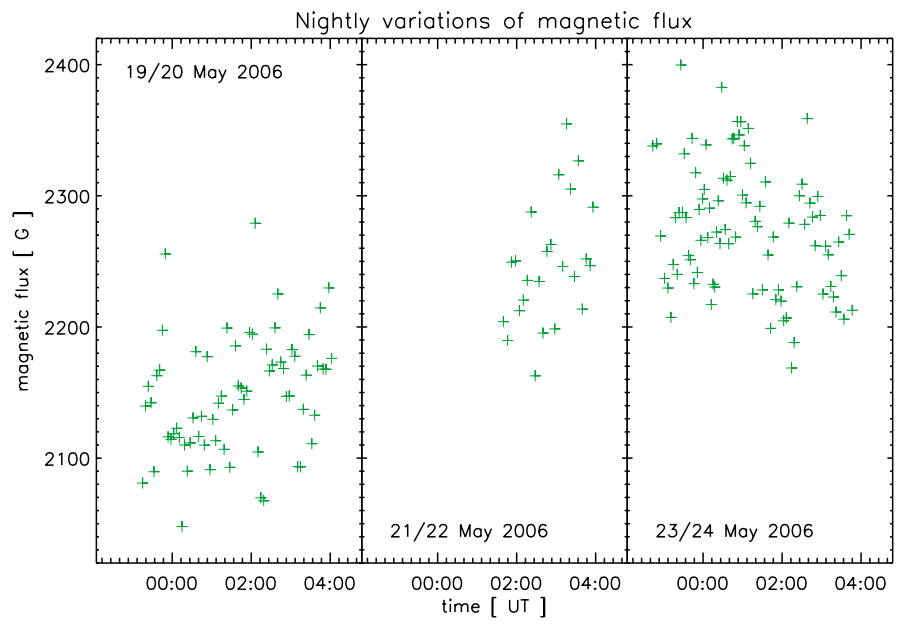

Fig. 1. Variation of the mean magnetic flux $B f$ over the three nights. The relative error on a an individual data point is about $80 \mathrm{G}$ (see text).

68, 24 and 89 spectra taken in our three half nights. The data were processed using the IDL based REDUCE reduction package (Piskunov \& Valenti 2002). Since the MIT/LL CCD-20 chip shows very strong fringing in the red wavelength region, we performed a 2D-flatfielding with standard calibration lamp exposures. The typical signal-to-noise ratio $(S N R)$ of a single spectrum is on the order of 40 around $1 \mu \mathrm{m}$.

\section{Method of analysis}

In order to determine the mean magnetic field strength in $\mathrm{CN}$ Leo we use the molecular $\mathrm{FeH}$ band near $1 \mu \mathrm{m}$; this band contains a set of narrow, individual absorption lines, some of which are magnetically very sensitive, while others are not. Unfortunately, so far no Landé g-factors are available for these lines. Therefore, magnetic flux measurements are performed by comparing $\mathrm{FeH}$ lines to spectra of stars with known magnetic flux and known activity, i.e., to template stars. The feasibility and performance of this method has been successfully demonstrated by Reiners \& Basri (2007). As a second measurement of the magnetic flux, we use the line ratio method suggested by Reiners \& Basri (2006) as a consistency check after we calculated the magnetic flux with the line-profile fitting method.

\subsection{Line profile fitting}

To determine the mean magnetic field of CN Leo we follow the approach by Reiners \& Basri (2007), who analyzed HIRES Keck data of 22 ultracool dwarfs. The basic idea of this method consists in modeling the observed spectrum of the target star as a linear combination of the spectrum of a magnetically inactive star with very low magnetic flux (we chose GJ 1002, M5.5) and that of a magnetically very active star (we chose G1 873, M3.5) with $B f \approx 3.9 \mathrm{kG}$ (Johns-Krull \& Valenti 2000). As actual templates we used the Keck HIRES spectra of Gl 873 and GJ 1002. A comparison of the UVES and Keck spectra of CN Leo shows that the resolving power of the HIRES and UVES spectra are very much alike. We therefore employed both data sets without any further assumptions on the instrument profiles.

Specifically, we construct a linear interpolation of the normalized spectrum $S_{\mathrm{CN} \text { Leo }}$ of CN Leo from the normalized template spectra $S_{\mathrm{Gl} 873}$ and $S_{\mathrm{Gl} 1002}$ through

$S_{\mathrm{CN} \text { Leo }}=p S_{\mathrm{Gl} 873}+(1-p) S_{\mathrm{GJ} 1002}$.
The weight $p$, with $0<p<1$, yields $B f=p B f(\mathrm{Gl} 873)=$ $p 3.9 \mathrm{kG}$. We find the best value of $p$ by $\chi^{2}$-minimization (Press et al. 1992) through a comparison of $S_{\mathrm{CNLeo}}$ with the actually measured (normalized) spectrum. Note that a few steps are required before the interpolation of the two template spectra can be computed: Both template spectra are adjusted to match the target spectrum in line depth and in the overall width of the magnetically insensitive lines (for more details, see Reiners \& Basri 2007). The widths of the magnetically insensitive lines are used to calibrate the line broadening that affects all spectral lines. This global broadening is dominated by stellar rotation and the spectrograph's resolving power. While stellar rotation can obviously be assumed constant during all observations, the spectral resolution can also be affected by the seeing conditions during observation. In a perfect spectrum, for a star with low rotation velocities this effect would mimic the effect of extra rotation and should show up if we formally allow the fit to also adjust the projected rotation velocity $v \sin i$ for each spectrum.

We performed the $\chi^{2}$-minimization first treating $v \sin i, B f$ (i.e., $p$, see Eq. (1)), and the line depth $a$ as free parameters. However, we did not find any correlation between $v \sin i$ and the seeing conditions that we measured through the widths of telluric lines. We therefore believe that small extra broadening due to variable seeing conditions does not significantly affect the line widths of the absorption lines, which are dominated by temperature and stellar rotation. Treating $v \sin i$ as a free parameter we found $v \sin i$ values between 2.0 and $2.5 \mathrm{~km} \mathrm{~s}^{-1}$, which are only marginally different given the comparably low resolution (1 resolution element $\left.\approx 7.8 \mathrm{~km} \mathrm{~s}^{-1}\right){ }^{1}$ Since the rotation velocity of CN Leo is not variable on the timescales of our measurements, we took the mean value of all values of $v \sin i$ from the fit, $\overline{v \sin i}=2.14 \mathrm{~km} \mathrm{~s}^{-1}$ (incidentally, the value of the mean coincides with the value of the median, so it is not necessary to decide which measure is best here). We then repeated the fit, now with a fixed value of $v \sin i=2.14 \mathrm{~km} \mathrm{~s}^{-1}$. This procedure did not significantly change the derived results when compared to the results achieved with $v \sin i$ as a free parameter. In particular, the derived $B f$ values do not vary significantly, and we proceed using the results achieved with the fixed value of $v \sin i$.

A total of 181 spectra of CN Leo were recorded during our campaign and were used to derive mean magnetic fields as described above. In Fig. 1, we plot all our $B f$ measurements versus time. The typical uncertainty of our $B f$ measurements is approximately $\overline{\Delta B f}=80 \mathrm{G}$; this value represents the $1-\sigma$ uncertainty from the $\chi^{2}$-fit, i.e., the values of $B f$ for which $\chi^{2}<\chi_{\min }^{2}+1$ (Press et al. 1992). We emphasize that this error is of purely statistical nature and cannot be identified as the true uncertainty of the absolute measurement of the magnetic flux $B f$. For the relative measurement, however, this is the uncertainty that we adopt. We note that our fitting procedure started with an initial guess of $B f=2500 \mathrm{G}$ for every spectrum, and that the algorithm did significantly modify this initial guess. We also tried the effect of a different but fixed value of $v \sin i$ and found that a different $v \sin i$ slightly modifies the absolute values of all measurements, but does not change our results on the differential measurements.

\subsection{Analysis of spectral differences}

Is there any true variation of $B f$ visible in our data? The difference in the magnetic flux between the individual spectra with

\footnotetext{
${ }^{1}$ Note that $v \sin i$ is the broadening required to match the template spectra and the spectra of CNLeo, thus it is only a lower limit for the true projected rotation velocity of CN Leo.
} 
Table 1. Mean magnetic flux, standard deviation, error of the nightly mean, number of observations per night for all three nights; the other columns contain results of non-parametric statistical tests (see text).

\begin{tabular}{ccccccc}
\hline \hline Night & $\overline{B f} \pm \sigma[\mathrm{G}]$ & $N$ & KS1 & KS2 & RS1 & RS2 \\
\hline 1 & $2148 \pm 45 \pm 5.5$ & 68 & - & - & - & - \\
2 & $2248 \pm 47 \pm 9.6$ & 24 & $7 \times 10^{-11}$ & - & 0 & - \\
3 & $2275 \pm 48 \pm 5.1$ & 89 & $3 \times 10^{-28}$ & $8.4 \times 10^{-3}$ & 0 & $7.7 \times 10^{-3}$ \\
\hline
\end{tabular}

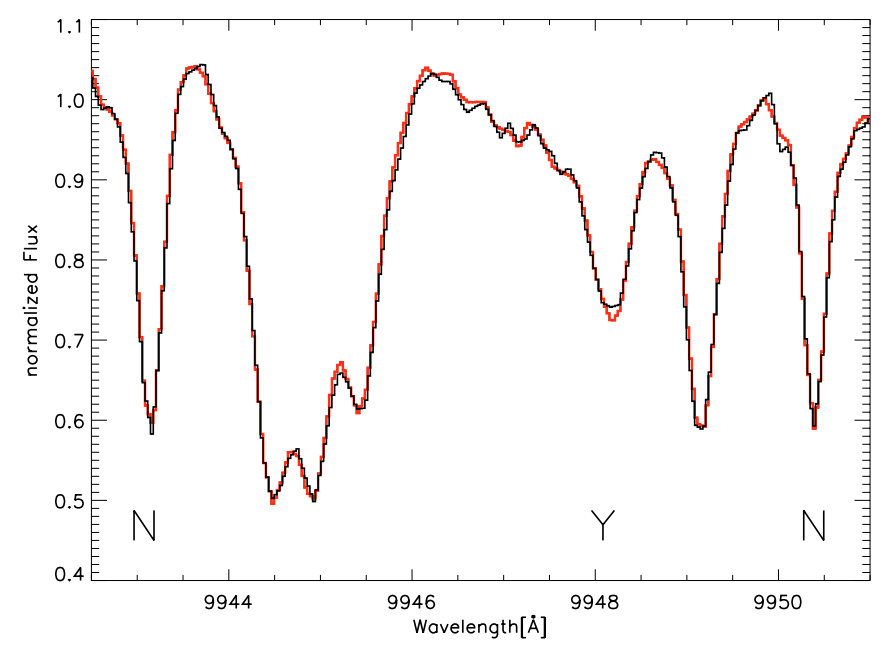

Fig. 2. Co-added spectra from the 68 spectra of the first night (red) and from the 89 spectra of the third night (black). At the achieved $S N R \approx$ 300 Zeeman broadening becomes visible in the most sensitive lines. Y: magnetically sensitive line, $\mathrm{N}$ : magnetically insensitive lines.

the maximum and the minimum flux is $\approx 350 \mathrm{G}$, i.e., less than $10 \%$ of the difference in flux between the two template stars of $\approx 3900 \mathrm{G}$. At the spectral type of CN Leo, the line depth of the magnetically most sensitive lines varies by $\approx 15 \%$ of the continuum value (e.g. the line at $9948 \AA$ varies between $\sim 0.58$ and $\sim 0.74$, cp. Fig. 6 in Reiners \& Basri 2007). Thus, in order to detect a magnetic flux variation of $350 \mathrm{G}$, a variation on the order of $1.5 \%$ has to be detected in this line, i.e., a $S N R$ on the order of 70 is required. Because of the short individual exposures such a $S N R$ is not available in our spectra, and therefore a direct comparison cannot be expected to yield a significant difference in the Zeeman signatures directly visible in the spectra. However, our $\chi^{2}$-fit uses simultaneously the information contained in many lines and hence is superior to a direct comparison of line depths.

Nonetheless, our $\chi^{2}$-fits could be fooled by a variety of effects that are difficult to control if the results cannot be independently verified. In order to achieve such a consistency check, we constructed two mean spectra by averaging all spectra from the first night (68) and all spectra from the third night (89). The mean values of $B f$ for the three different nights are given in Table 1 . Between the first and the third night $\overline{B f}$ differs by $125 \mathrm{G}$. The summation of all spectra in the first and third night with a $S N R$ of typically $\sim 40$ each yields average spectra with a $S N R$ on the order of $\sim 300$. Since a $S N R$ on the order of 200 is required to detect a difference of $125 \mathrm{G}$ in the $9948 \AA$ lines between the two mean spectra (see above), the difference in the magnetically most sensitive lines should become visible in such a comparison.

In Fig. 2, we show the mean spectra for the first and third nights. The magnetically very sensitive line at $9948 \AA$ is marked with a "Y", two relatively insensitive lines are marked with an "N". As expected from our estimate, a small difference becomes
Table 2. Line depth ratios as introduced in Reiners \& Basri (2006). Both ratios are consistent with our results (see text).

\begin{tabular}{lcc}
\hline \hline & $9949.11 / 9948.13$ & $9956.77 / 9958.25$ \\
\hline$B f_{\text {Night1 }}$ & $1.48 \pm 0.01$ & $1.44 \pm 0.01$ \\
$B f_{\text {Night3 }}$ & $1.59 \pm 0.01$ & $1.45 \pm 0.01$ \\
\hline
\end{tabular}

visible in the magnetically sensitive line, while insensitive lines do not show systematic variation.

\subsection{Magnetically sensitive line ratios}

As a second consistency check we use the two mean spectra for the first and third nights and calculate the two line depth ratios as suggested by Reiners \& Basri (2006). Each ratio is calculated from measuring the depth of two neighbored lines, one of which is Zeeman sensitive and one is insensitive. We use the two ratios 9949.11/9948.13 and 9956.77/9958.25 as in Reiners \& Basri (2006), both ratios should be larger at high magnetic flux. The results are given in Table 2 and should be compared to Fig. 7 of Reiners \& Basri (2006). That figure shows that indeed the $9949.11 / 9948.13$ ratio is more sensitive to magnetic flux variation than the $9956.77 / 9958.25$ ratio is. Both ratios and their variation are in good agreement with our results from $\chi^{2}$-fitting.

\section{Results, discussion and conclusions}

As a first result of our analysis of 181 spectra of CN Leo we note that magnetic flux is consistently detected in each UVES spectrum. At first sight the data are consistent with the assumption of a constant magnetic flux during each night, while the magnetic flux does appear to vary from night to night. In Table 1, we give the derived mean values of $B f$ and their standard deviation for all three nights. The standard deviation is consistent with the estimated uncertainty of an individual observation; in fact, it is a even little smaller, which could mean that the differential measurements are somewhat more precise than estimated.

The uncertainty in the absolute value of the field is dominated by our ignorance of the splitting patterns of the magnetically sensitive $\mathrm{FeH}$ lines. Our method only employs a linear interpolation between the two spectra of the template stars, i.e., it assumes that the splitting patterns are the same ( $B$ is constant), and that only the filling factor $f$ is variable. This is probably not the case, although the change in filling factor is usually assumed to be the dominant factor. At any rate, we cannot yet calculate different splitting patterns and thus, following Reiners \& Basri (2007), we estimate the uncertainty on the absolute value to be on the order of a few hundred Gauss. However, here we are interested in the accuracy of the absolute measurements only to a lesser extent, rather are we interested in the differential measurements comparing the values of $B f$ during several nights and within a given night. Since the magnetically insensitive lines stay constant, they provide a very accurate calibration, particularly when intercomparing the exposures. Thus, we estimate that our uncertainties in the individual differential measurements are indeed on the order of the uncertainty of the $\chi^{2}$-fit, i.e., $80 \mathrm{G}$.

Inspecting the nightly mean magnetic field values and their dispersion (Table 1), the difference in these means appears to be significant only on the $1-2 \sigma$ level when compared to the variance of the distribution. We checked the nature of the distribution of our nightly measurements around the respective nightly means and found very good agreement with a Gaussian 


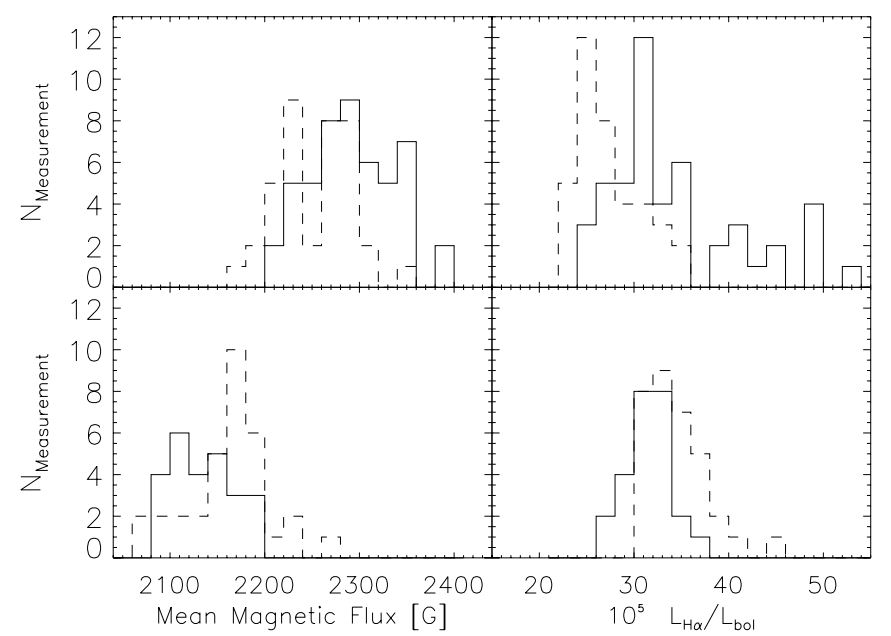

Fig. 3. Magnetic flux (left panels) and $\mathrm{H} \alpha$ emission (right panels) for first night (lower panels) and third night (upper panels); in each panel, data for the respective (half-)nights are shown for the first half (solid histograms) and second half (dashed). Note the correlated change in magnetic flux and $\mathrm{H} \alpha$ emission during the first and third half night. Eight data points in a large flare during the first half of the first night are not shown because of saturation of the $\mathrm{H} \alpha$ line core.

distribution and therefore the accuracy of the mean ought to be much smaller than the width of the observed distribution. Computing the uncertainty of the nightly means, we find values reduced by $\sqrt{N}$ (cf., Table 1 ) and the differences between the individual become very significant indeed. We verified the existence of night-to-night magnetic field variations also by analyzing the actually measured distributions of $B f$ using two nonparametric test methods. We specifically applied the Wilcoxon rank sum test (denoted by RS in Table 1) and the two sample Kolmogorov-Smirnov test $(\mathrm{KS})$ to investigate whether the measured $B f$ during our three observing nights are drawn from the same parent population (KS1 and RS1 indicate comparison to the first night, KS2 and RS2 to the second). The derived Probabilities from the KS- and RS-tests that the null hypothesis "no night-to-night magnetic flux change" is correct are also listed in Table 1. As is clear from Table 1, the field measurements for the nights 1 and 2 and 1 and 3 differ in an extremely significant fashion, and even the measurements between nights 2 and 3 appear to differ at a confidence level in excess of 0.99 independent of the test statistics used. In summary, the above statistical analysis strongly suggests magnetic flux changes on time scales of 48 hours.

How about the presence of flux changes on shorter time scales? In order to address this issue we subdivided the available magnetic flux measurements for each of our three nights into two halves and applied the same tests performed for night-tonight variability to assess any intra-night variability. While we cannot claim any intra-night variability during night 2 (with only $24 B f$ measurements available), we find significant intra-night variability for night 1 and night 3 . The variability is such that the magnetic flux during night 1 appears to be increasing, while it is decreasing during night 3 . Interestingly, the intra-night variability in chromospheric emission lines follows the same pattern as the magnetic flux. We measured the chromospheric flux from the $\mathrm{H} \alpha$ lines contained in our spectra (see Reiners \& Basri 2007) and calculated $L_{\mathrm{H} \alpha} / L_{\mathrm{bol}}$ with the same value of $L_{\mathrm{bol}}$ for all spectra. The change in magnetic flux and $\mathrm{H} \alpha$ emission is directly visible in the distribution of magnetic flux and $\mathrm{H} \alpha$ emission during the first and second quarters of nights 1 and 3 (first and second half of our observation in each night). The distribution for nights 1 and 3 are shown in the lower and upper panels of Fig. 3, respectively. We find the difference between the two distributions shown in each panel of Fig. 3 is statistically highly significant.

These trends are verified by formally describing the nightly magnetic flux measurements as a linear function with time. Linear regression analysis shows that including a linear trend significantly better describes the data as opposed to the assumption of a constant magnetic flux; the values of $\Delta \chi^{2}$ are 6.0 and 7.3 for nights 1 and 3 respectively. We find a positive flux change during night 1 and a negative change during night 3 ; the observed flux changes to an amount of $\approx 50 \mathrm{G}$ over a time scale of 6 hours. While we cannot entirely exclude artifacts of the fit procedure as the cause for the intra-night variations, two facts make us confident that the observed changes are of a physical origin: First, the observed changes are of opposite sign during night 1 and 3. Second, the magnetic flux changes during those nights correlate with corresponding activity changes during the same nights, which we have verified in the $\mathrm{H} \alpha$ lines contained in our spectra (see Fig. 3). We note that our spectra contain a multitude of chromospheric emission lines, which are correlated with $\mathrm{H} \alpha$ emission. These will be analyzed in a subsequent publication.

Reiners \& Basri (2007) measured $B f=2.4 \mathrm{kG}$ in a spectrum of CN Leo taken in 2005. This result is on the upper end of the values we found during our observations. However, their spectra were not taken at the same instrument, which introduces uncertainties due to the different calibrations. Hence their result is consistent with our measurements.

In summary, we have successfully measured a mean magnetic field of $B f \approx 2.2 \mathrm{kG}$ on the flare stars CN Leo using the FeH bands near $1 \mu \mathrm{m}$. Our monitoring campaign shows definite magnetic flux changes of $\sim 100 \mathrm{G}$ on a time scale of 48 hours. Further, the data of the individual (half) nights suggest flux changes on even shorter time scales, which are correlated with the general activity level of $\mathrm{CN}$ Leo as measured in $\mathrm{H} \alpha$ emission. Further monitoring of magnetic fields and their variations in CN Leo and similar stars appears very promising.

Acknowledgements. We thank Prof. G. Basri for letting us use his Keck spectra of GJ 1002 and G1 873, and the referee, Christopher Johns-Krull for a very constructive report. AR has received research funding from the European Commission as an Outgoing International Fellow (MOIF-CT-2004-002544). CL acknowledges financial support from DLR under 50OR0105.

\section{References}

Donati, J.-F., Forveille, T., Cameron, et al. 2006, Science, 311, 633 Fuhrmeister, B., Liefke, C., \& Schmitt, J. H. M. M. 2007, A\&A, accepted Fuhrmeister, B., Schmitt, J. H. M. M., \& Hauschildt, P. H. 2005, A\&A, 439, 1137

Henry, T. J., Subasavage, J. P., Brown, M. A., et al. 2004, AJ, 128, 2460

Johns-Krull, C., \& Valenti, J. A. 2000, ASPC, 198, 371

Kirkpatrick, J. D., Henry, T. J., \& McCarthy, D. W. 1991, ApJS, 77, 417 Mohanty, S. \& Basri, G. 2003, ApJ, 583, 451

Pavlenko, Y. V., Jones, H. R. A., Lyubchik, Y., Tennyson, J., \& Pinfield, D. J. 2006, A\&A, 447, 709

Piskunov, N. E. \& Valenti, J. A. 2002, A\&A, 385, 1095

Press W. H., Teukolsky S. A., Vetterling W. T., \& Flannery B. P. 1992, Numerical Recipes in C (Cambridge Universtity Press)

Reid, I. N., Hawley, S. L., \& Gizis, J. E. 1995, AJ, 110, 1838

Reiners, A., \& Basri, G. 2006, ApJ, 644, 497

Reiners, A., \& Basri, G. 2007, ApJ, 656, 1121 